\title{
COMPORTAMIENTO HIDROLÓGICO DE LA CUENCA ALTA DEL ARROYO PIGÜÉ (BUENOS AIRES, ARGENTINA): BALANCE HÍDRICO (1964-2007)
}

\author{
Patricia Rosell ${ }^{1}$, Belén García Martínez ${ }^{2}$ \\ ${ }^{1}$ Dpto. de Geografía y Turismo, Universidad Nacional del Sur (Bahía Blanca, Argentina) \\ ${ }^{2}$ Dpto. de Geografía Física y A.G.R., Universidad de Sevilla (Sevilla, España)
}

\section{RESUMEN}

En este trabajo se aborda el estudio hidrológico de la cuenca alta del Arroyo Pigüé, a través del estudio de la serie de precipitaciones y el balance hídrico del suelo de la cuenca. Desde un punto de vista climático, se constata la existencia de un ciclo húmedo en la región, a partir de la década de los setenta, que se traduce, a nivel hidrológico en una progresiva sobresaturación de los suelos, con fuertes descargas en dirección a la red de drenaje del Pigüé. Ésta, parcialmente desconectada en el territorio, por la intensificación de las prácticas agrícolas, impide la evacuación encauzada hacia la localidad, agravando más si cabe, el riesgo de inundación en periodos extremadamente húmedos.

Palabras clave: Balance hídrico, Hidrología, Pigüé, Buenos Aires, Argentina.

\section{RÉSUMÉ}

À ce travail on aborde l'étude hydrologique du haut bassin du Ruisseau Pigüé, à travers de l'étude de la série de précipitations et le bilan hydrique du sol du bassin. D'un point de vue climatique, on constate l'existence d'un cycle humide dans la région, à partir de la décennie des soixante-dix que l'on traduit, à un niveau hydrologique dans un sobresaturación progressif des sols, avec fortes décharges dans la direction du réseau de drainage du Pigüé. Par l'intensification des pratiques agricoles, celle-ci, partiellement déconnectée dans le territoire empêche l'évacuation dirigée vers la municipalité, en aggravant plus, le risque d'inondation dans des périodes extrêmement humides.

Mots clefs: Bilan hydrique, Hydrologie, Pigüé, le Buenos Aires, l’Argentine. 


\section{Introducción y metodología}

La necesidad de conocer la capacidad de retención hídrica del suelo, así como el comportamiento que adquiere a lo largo del año hidrológico, son aspectos ampliamente reconocidos en el ámbito de la Hidrología (Thornthwaite y Matter 1957; Grayso et al., 1997; Famiglietti et al., 1998; Bárdossy y Lhemann, 1998; Cosandey et Robinson, 2007). Su influencia en los procesos hidrológicos en general, condiciona forzosamente el funcionamiento hidrológico de los sistemas fluviales, a escala de cuencas vertientes, en la medida en la que asegura el caudal fluvial: en primer lugar, generando y concentrando la escorrentía, en fase de ladera, y en segundo lugar, influyendo en el régimen hídrico y las crecidas fluviales, mediante la descarga de los acuíferos, ya en la fase propiamente fluvial.

Sobre esta base, uno de los principales factores responsables de la escorrentía es precisamente el estado de humedad del suelo. En este sentido, los autores anglosajones distinguen dos tipos de flujos: el generado por superarse el umbral de infiltración, en un suelo no saturado y en un suelo saturado. Esta clasificación ha permitido reagrupar en la primera categoría la escorrentía desarrollada en las vertientes, la cual se encuentra desconectada de los cursos fluviales. Mientras que en la segunda, el caudal fluvial no depende exclusivamente del estado hídrico medio de la cuenca, sino del estado de las reservas hidrológicas, asegurando el caudal fluvial también en periodos de estiaje y avenidas fluviales.

En sistemas fluviales donde se carecen de registros foronómicos, para el estudio del comportamiento hidrológico fluvial, el análisis de otros parámetros a nivel de cuenca se hace imprescindible. Con carácter general, los trabajos sobre morfometría o Hidrografía, en el seno de la Geografía, se orientan precisamente al estudio hidrológico a partir de la definición morfométrica de su cuenca y su red de drenaje, como respuesta de los ríos a la descarga hídrica y/o a las modificaciones que experimenta su entorno. De hecho, estudios previos en la zona de estudio (Zinger y Rosell, 2004) señalan como factores determinantes de las inundaciones, en el municipio de Pigüé, los de tipo morfométrico, como la forma y el tamaño de la cuenca superior o las pendientes acentuadas; con otros de tipo climáticos y/o ambientales, como puede ser los cambios de usos del suelo.

Este trabajo pretende aportar, precisamente, claves en este sentido, que ayuden a la interpretación general del comportamiento hidrológico de la cuenca alta del Pigüé. Para ello se establecen los siguientes objetivos: analizar, por un lado, las condiciones climáticas de la segunda mitad del siglo XX y comienzos del siglo XXI; y caracterizar, por otro, el comportamiento hídrico del suelo, a través de la elaboración de un balance hídrico característico de las condiciones ambientales de la cuenca.

El balance hídrico es un método de cálculo que se utiliza en distintas disciplinas relacionadas con temas hidrológicos. En Geografía es de suma utilidad y su aplicación en temas climáticos e hidrográficos facilita la interpretación de los procesos comprendidos en el ciclo hidrológico. Así mismo, el estudio del balance hídrico local y regional, es una de las principales fuentes de análisis y explicación de las variaciones en el tiempo y en el espacio, no sólo de las condiciones climáticas sino también de sus repercusiones en el régimen hidrológico (Campo de Ferreras et al., 2004). Por tanto, el cálculo del balance hídrico explica lo que ocurre con la precipitación que cae en una cierta área, con el fin fundamental de separar dos fracciones: la que es devuelta a la atmósfera sin ninguna posibilidad de alimentar a las corrientes superficiales y/o subterráneas y aquella otra que escapa a los procesos de evaporación y transpiración, y puede ser abastecedora de los recursos hidráulicos superficiales y/o subterráneas y generadora de los caudales de avenida. 
Para ello, utilizaremos el método propuesto por Thornthwaite y Matter (1957), con la finalidad de establecer relaciones de reciprocidad con los procesos hidrológicos extremos (avenidas) en la cuenca alta del Arroyo Pigüé, a partir de la disponibilidad de agua por saturación del suelo, en la serie 1964-2007.

\section{2. Área de estudio}

La cuenca superior del arroyo Pigüé se localiza en el Sudoeste de la Provincia de Buenos Aires, en el Distrito de Saavedra (República Argentina) aguas arriba del núcleo de población que le da nombre (Fig. 1).

La región se enmarca en la porción meridional de la gran llanura Chaco Pampeana, cuenca sedimentaria que se depositó sobre los pilares del macizo de Brasilia. Conforman la geomorfología regional los cordones occidentales del Sistema de Ventania, y una llanura que bordea los faldeos serranos, recorrida por arroyos que divergen de los mismos.

El Sistema de Ventania es un plegamiento Paleozoico cuarcítico fuertemente diaclasado que, en forma de arco, tiene una extensión de $170 \mathrm{~km}$. y un ancho máximo de $50 \mathrm{~km}$. Su mayor altitud sobrepasa los 1200 m s.n.m. La repercusión tectónica provocada por el ascenso de los Andes durante el Terciario, se manifiesta en diferentes sistemas de fallas regionales que afectaron parte del sudoeste bonaerense. Como resultado, el sistema serrano queda enmarcado entre dos fallas paralelas secundarias que controlan el relieve general y el comportamiento de los escurrimientos, determinando el diseño y la distribución de las cuencas de drenaje. El grado de diaclasamiento define la porosidad secundaria y el comportamiento de la infiltración de las aguas superficiales y subterráneas.

Cerrando la secuencia estratigráfica, se dispone un manto moderno de desigual espesor de cronología Holocena, denominado grupo Postpampeano. Éste, conforma extensos mantos de loess de significación ambiental muy directa sobre la formación de los suelos, con un nivel de cementación calcárea, la tosca, que muchas veces aflora en lomadas de interfluvios (González Uriarte, 1988).

En este contexto litoestructural, el arroyo Pigüé, rodeado por cerros cuyos alturas no sobrepasan los $600 \mathrm{~m}$., drena las vertientes del piedemonte serrano con una pendiente media del 3.5\%, hasta desembocar en la depresión tectónica del Lago Epecuén, tras 84 $\mathrm{km}$ de recorrido. Ha sido catalogado como pequeño y tranquilo a tenor de su longitud y su caudal modular, estimado en 1,5 m³/s (Rosell y Zinger, 2004). En cuanto a su cuenca alta, de algo mas de $114 \mathrm{~km}^{2}$, está definida de manera general por su forma próxima al círculo (Rosell y Zinger, 2003) y una red de drenaje de tipo dendrítico de orden 3 y relación de bifurcación próxima al 3 , actualmente desconectada por las intensas labores agrícolas.

Así mismo, es de destacar, en su comportamiento hidrológico, los eventos de crecidas registrados en la zona, por los que se ha visto directamente afectado el municipio de Pigüé. En estos episodios, el caudal adquiere carácter turbulento y peligroso, durante periodos más o menos breves, en coincidencias con precipitaciones copiosas en su cuenca superior; lo que puede darnos una idea del comportamiento irregular del régimen fluvial del arroyo Pigüé. Así, tributarios que permanecen sin actividad alguna en periodos secos, recobran su funcionalidad durante estos episodios de avenidas, evacuando una gran cantidad de aluviones hacia el cauce principal (Zinger y Rosell, 2004).

Desde un punto de vista climático, el área queda incluida dentro de la faja zonal de clima templado subhúmedo húmedo. Si bien la isohieta de $750 \mathrm{~mm}$ expresa una moderada precipitación, existe un alto grado de variabilidad en los registros pluviométricos anuales que explican las alternancias de ciclos húmedos y secos. Las mayores concentraciones se 

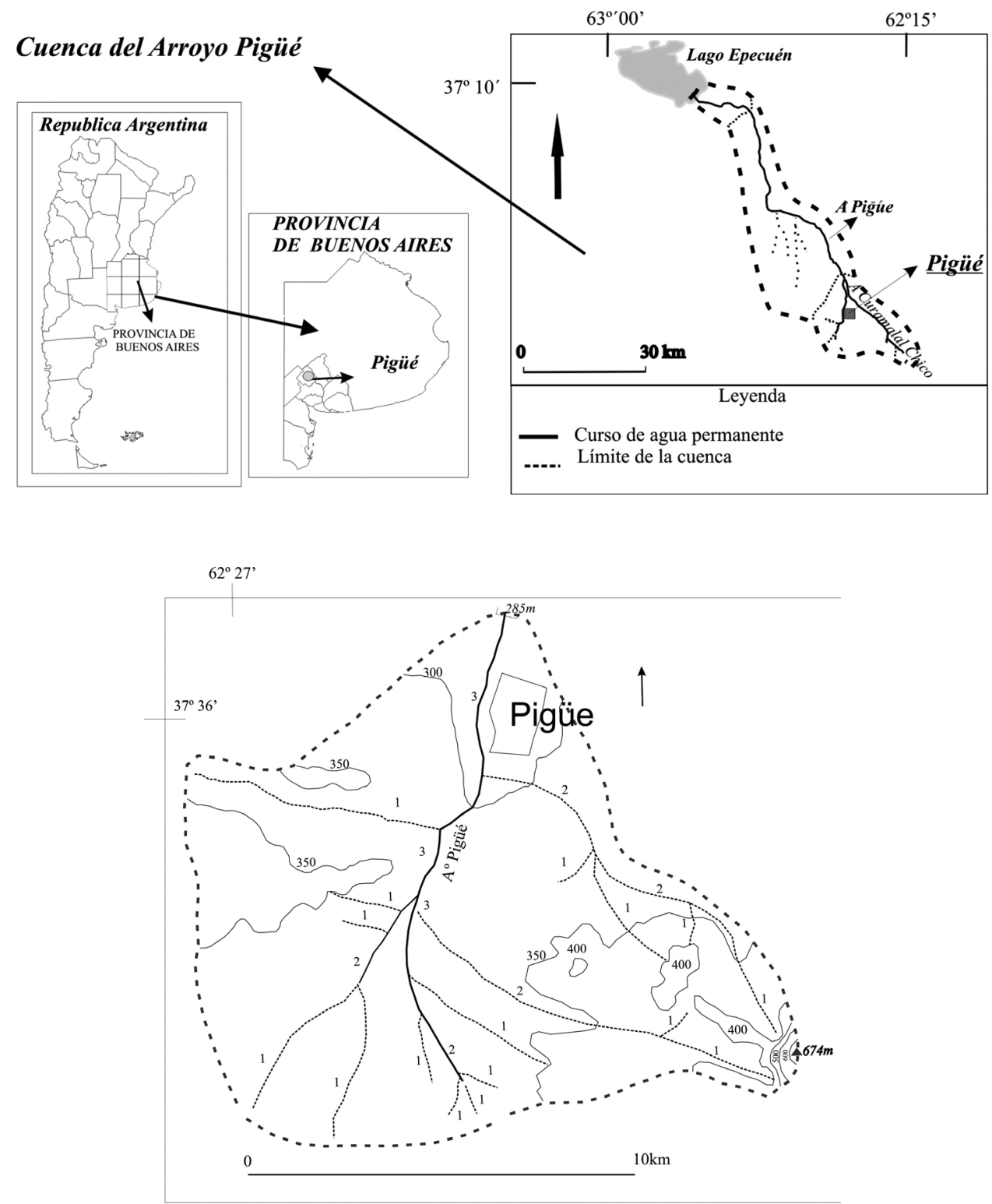

Figura 1. Localización de la cuenca alta del Arroyo Pigüé.

dan en las estaciones de primavera y otoño, específicamente en los meses de octubre y mayo. En cuanto a las temperaturas, los valores anuales medios oscilan entre $14^{\circ} \mathrm{C} \mathrm{y} 20^{\circ} \mathrm{C}$. En los meses más cálidos los valores extremos superan los $40^{\circ} \mathrm{C}$ y durante el invierno, la influencia del ingreso de olas de frío polar genera un descenso de las temperaturas a valores extremos bajo cero (Capelli y Campo, 2004). 
Bajo las condiciones del clima mencionado tienen amplia difusión los Udoles, suelos Molisoles que responden a un régimen único, entre 60 y 90 días secos al año. Reúnen estas características, según la clasificación del Atlas de Suelo de la República Argentina (1971), los siguientes suelos:

a) Hapludoles líticos y en algunos casos petrocálcicos, que se desarrollan en el ambiente periserrano, originados a partir de los depósitos del loess. Son suelos de textura franco limosa a franco arcillo-limosa. Su espesor llega a casi un metro con permeabilidad moderada y buena materia orgánica.

b) Argiudoles típicos que cubren totalmente el faldeo de las sierras, con un espesor que alcanza el metro. Poseen un horizonte enriquecido con arcillas de iluviación, no muy espeso y que decrece rápidamente en profundidad. De buena textura y estructura superficial, potenciada por el nivel de materia orgánica, éstos presentan una adecuada retención de humedad, aún cuando encuentran en la tosca ciertos factores limitantes.

En estos suelos y en función de las características topográficas predominantes en la zona, se desarrolla como expresión natural, el pastizal pampeano; el cual se encuentra escasamente representando, en la actualidad, a partir de la cota de $400 \mathrm{~m}$, donde aflora, precisamente, la roca cuarcítica intensamente diaclasada.

En definitiva, estas características geoambientales exponen condiciones ecológicas favorables para un uso de suelo apto para la actividad agropecuaria. Así, y desde que un grupo de colones franceses fundaran la colonia agrícola de Pigüé a finales del siglo XIX, el cultivo del trigo ha sido hasta el presente, el sustento para su desarrollo económico. Es precisamente este hecho, el que plasma el patrimonio histórico-cultural de Pigüé y su entorno, quedando expresamente en el territorio, los vínculos y las relaciones intrínsecas entre naturaleza y sociedad. Como herencia de esa primera apropiación del medio predomina hasta hoy un parcelamiento rural regular, de diseño damero, cuyas unidades de explotación varían entre 150 y 500 has.

\section{Caracterización hidroclimática de la Cuenca del Arroyo Pigüé}

\subsection{Serie de precipitaciones 1888-2007}

Se analizan las series de precipitación publicadas por el Servicio Meteorológico Nacional (SMN) y por la Cooperativa Eléctrica Pigüé, para el período 1888-2006. Durante esta serie de 119 años, el promedio de precipitaciones es de 750mm; detectándose marcadas oscilaciones entre valores superiores e inferiores a la media, con una tendencia al incremento hacia la última década del siglo XX (Figura 2).

En ella, se identifica dos periodos, uno seco y otro húmedo, que se corresponden con los resultados obtenidos en las investigaciones de autores como Scian y Donnari (1997), Canziani (2003), Aiello (2004) y Kruse y Laurencena (2005), en la región pampeana.

El ciclo seco en la región de Pigüé queda definido entre 1888 y 1972, cuando la mayor parte de los registros se sitúan por debajo de la media de $750 \mathrm{~mm}$. En esta fase que abarca un total de 84 años, 61 de ellos no superaron la media y siete de ellos, 1896, 1897, 1898, 1909, 1910, 1924 y 1962 fueron extremadamente secos con guarismo que no superaron los $350 \mathrm{~mm}$. Concretamente, en el año 1962 se registró el menor valor de precipitaciones en Pigüé, al no superar los 240mm; valor que por otro parte, mantiene una estrecha vinculación con la gran sequía que afectó a la Argentina en ese mismo año. Sólo 23 años superaron 


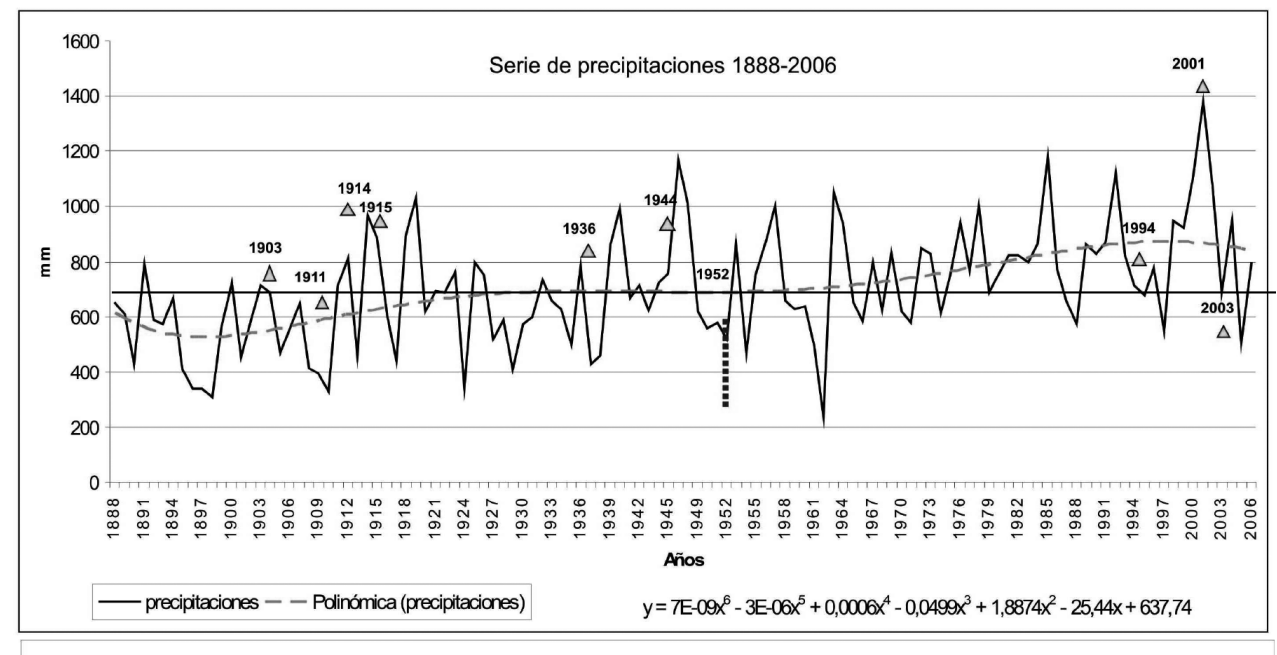

$\triangle$ Inundaciones $\quad$............ Construcción del canal derivador

Figura 2. Variabilidad interanual de las precipitaciones en Pigüé entre1888 y 2006 (A partir de los datos proporcionados por la Cooperativa Eléctrica de Pigüé y el SMN). Se incluye las principales inundaciones acontecidas y la puesta en funcionamiento del Canal Derivador.

la media de precipitaciones en este periodo seco, alcanzándose guarismos superiores a los 1000 mm en los años 1919, 1946, 1948, 1957 y 1963. En síntesis, el 72,62\% de esta fase seca registró precipitaciones por debajo de la media con eventos de sequías. Se corresponde este análisis con la investigación de Scian y Donnari (1997), en la que se establece una reducción de estos eventos extremos secos hacia el año 1972 en gran parte del sudoeste de la región pampeana.

A partir del año 1973 y hasta el año 2006 se registra un considerable aumento de las lluvias, sin que se detecte ningún evento severo de sequía en la región. En esta fase de 33 años, un total de 24 superaron la media de $750 \mathrm{~mm}$, representando el $75 \%$ del período considerado. Por otro lado, los años 2000, 2001 y 2002 fueron tres años consecutivos con registros mayores a los $1000 \mathrm{~mm}$. Todo ello, define una fase húmeda o ciclo húmedo al final de la serie, que afecta a toda la región pampeana. En este contexto regional, investigaciones llevadas a cabo por Hoffman (1987), quien precisa el desplazamiento de la isohietas aproximadamente $200 \mathrm{kms}$ al Oeste; o por Kruse y Laurencena (2005), advierten que en la Provincia de Buenos Aires a principios de la década de los setenta, se inicia un ciclo húmedo que se prolonga mas allá del año 2000, caracterizado por el aumento de las precipitaciones que superan los registros históricos del siglo XX. Por otro lado, Aiello (2004), con la comparación de la distribución de las lluvias medias anuales para los períodos 1941-70 y 1971-2004, detecta el incremento de los valores medios de precipitación anual en toda la provincia de Buenos Aires. Este incremento estimado por el autor, asciende a $150 \mathrm{~mm} / \mathrm{año}$ en el Noroeste de la Provincia, y a 100mm/año en el Distrito de Saavedra, donde se localiza el municipio de Pigüé.

En definitiva, con todo ello puede argumentarse un ciclo húmedo en la región, producido por un desplazamiento histórico de las isohietas hacia el Sur y el Oeste a partir del año 
1970, que conlleva una reducción en la frecuencia de las sequías, características del ciclo seco, y un aumento en el volumen de las precipitaciones.

\subsection{Principales eventos de avenidas y su correlación con eventos de precipitaciones de carácter tormentoso}

En este periodo de ciento dieciocho años, en el que no existe ningún tipo de control de aforos en la zona, el análisis hidrológico se ha podido efectuar mediante el registro histórico de crecidas del arroyo Pigüé en su tramo alto. Dicho análisis se realiza a través de las referencias relevadas en la presa local (Museo Regional Pigüé), que permite establecer, cualitativamente, las dimensiones de dicho fenómeno; y a partir de los registros meteorológicos recogidos en la Estación Meteorológica de Pigüé, desde su fundación en el año 1934.

El estudio de las inundaciones en la zona permite clasificarlas, de manera general, en recurrentes y extraordinarias, en función de la magnitud y los daños que generan en el municipio de Pigüé. En cualquier caso, los eventos de lluvias torrenciales asociados a las inundaciones quedan recogidos en la prensa local, con referencia al carácter torrencial de la precipitación, a los anegamientos en el municipio, al volumen de aluviones que transportan las torrenteras, a los caminos rurales convertidos en vías de escurrimiento, o a los desbordamientos del arroyo Pigüé.

De esta manera, se definen como recurrentes aquellas que afectaron tan sólo a los sectores topográficamente más bajos de la ciudad, sin implicar importantes daños o pérdidas. En estos casos, son numerosas las referencias a los desbordamientos locales del arroyo que provocan el anegamiento de caminos, la destrucción de terraplenes o las vías de ferrocarril. Entre ellas, se encuentran las inundaciones acontecidas en los años 1914, 1930, 1933, 1936, 1944, 1945, 1996, 1997, 2001, 2002 y 2005. Por su parte, las extraordinarias son consideradas aquellas a las que se les suma el caudal desbordado del arroyo Pigüé, asociado a lluvias torrenciales en la cabecera y que conllevan pérdidas de vidas humanas y numerosos daños materiales. Son consideradas extraordinarias las que tuvieron lugar en los años 1903, 1911, 1914, 1915 y 2003.

A efectos de correlacionar las inundaciones con eventos torrenciales de precipitación, dado la ausencia de datos hidrológicos aforados, se analizan los registros de ocho estaciones meteorológicas en la región de la Pampa bonaerense, incluida la localizada en el municipio de Pigüé. En todas ellas, quedan registradas, para los periodos 1911-1970 y 1980-2000, precipitaciones consideradas extremas, por su volumen de precipitación en cortos periodos de tiempo y que grosso modo se clasifican en tres rangos (Canziani, 2003): tormentas $\geq$ $60 \mathrm{~mm}, \geq 80 \mathrm{~mm}, \geq 100 \mathrm{~mm}$.

Tal y como puede observarse en la figura 3 , los guarismos $\geq 60 \mathrm{~mm}$ se incrementan, de manera general, en el periodo 1981-2000; detectándose un incremento destacado, en el número de casos, en la estación de Pigüé. Del mismo modo, los histogramas correspondientes al número de tormentas superiores a 80 y 100mm, manifiestan un aumento de los registros en todas las estaciones para el periodo 1981-2000. Sin embargo, es significativo el incremento cercano al $100 \%$ en el número de tormentas, sea cual sea su rango, en las localidades de Bolívar, Coronel Suárez, Balcarce y Pigüé.

Atendiendo especialmente a la estación de Pigüé, se constata un incremento del número de tormentas, para los tres rangos, en el segundo periodo; incremento que en términos generales supone el $100 \%$ respecto al periodo 1911-1970. Si comparamos este comportamiento con el de la serie de precipitaciones anuales, descrita anteriormente, se infiere una relación ciclo seco con una disminución del número de eventos tormentosos y ciclo húmedo con el aumento de dichos eventos. 
De esta manera, durante el periodo 1911-1970 se registran más de 40 casos de lluvias torrenciales $\geq 60 \mathrm{~mm}, 15$ casos $\geq 80 \mathrm{~mm}$ y 10 casos $\geq 100 \mathrm{~mm} ; 65$ eventos en cincuenta y nueve años. Por su parte, durante este intervalo tienen lugar en Pigüé cuatro inundaciones de carácter extraordinario (1903, 1911, 1914 y 1915); de las que no existen registros de la lluvia caída, aunque sí referencias en la prensa local de las fuertes avalanchas de agua provenientes del sistema serrano. Mientras que para el periodo 1980-2000 se registraron 80 casos de lluvias torrenciales $\geq 60 \mathrm{~mm}, 42$ casos $\geq 80 \mathrm{~mm}$ y 23 casos $\geq 100 \mathrm{~mm}$, esto es, 145 eventos tormentosos tan sólo en diecinueve años. Y ninguna inundación de carácter extraordinario.

Esta particularidad tiene, sin duda, su razón de ser en la puesta en funcionamiento de un Canal Derivador en el año 1952. Dicho canal, abierto al Sur de la ciudad de Pigüé, tiene como objetivo la función de evacuar hacia el tramo principal del arroyo Piguié las aguas de escurrimiento provenientes de la cabecera. En este sentido, cabe pensar que la construcción del canal haya mitigado los efectos de las avalanchas y minimizado el problema de las inundaciones.
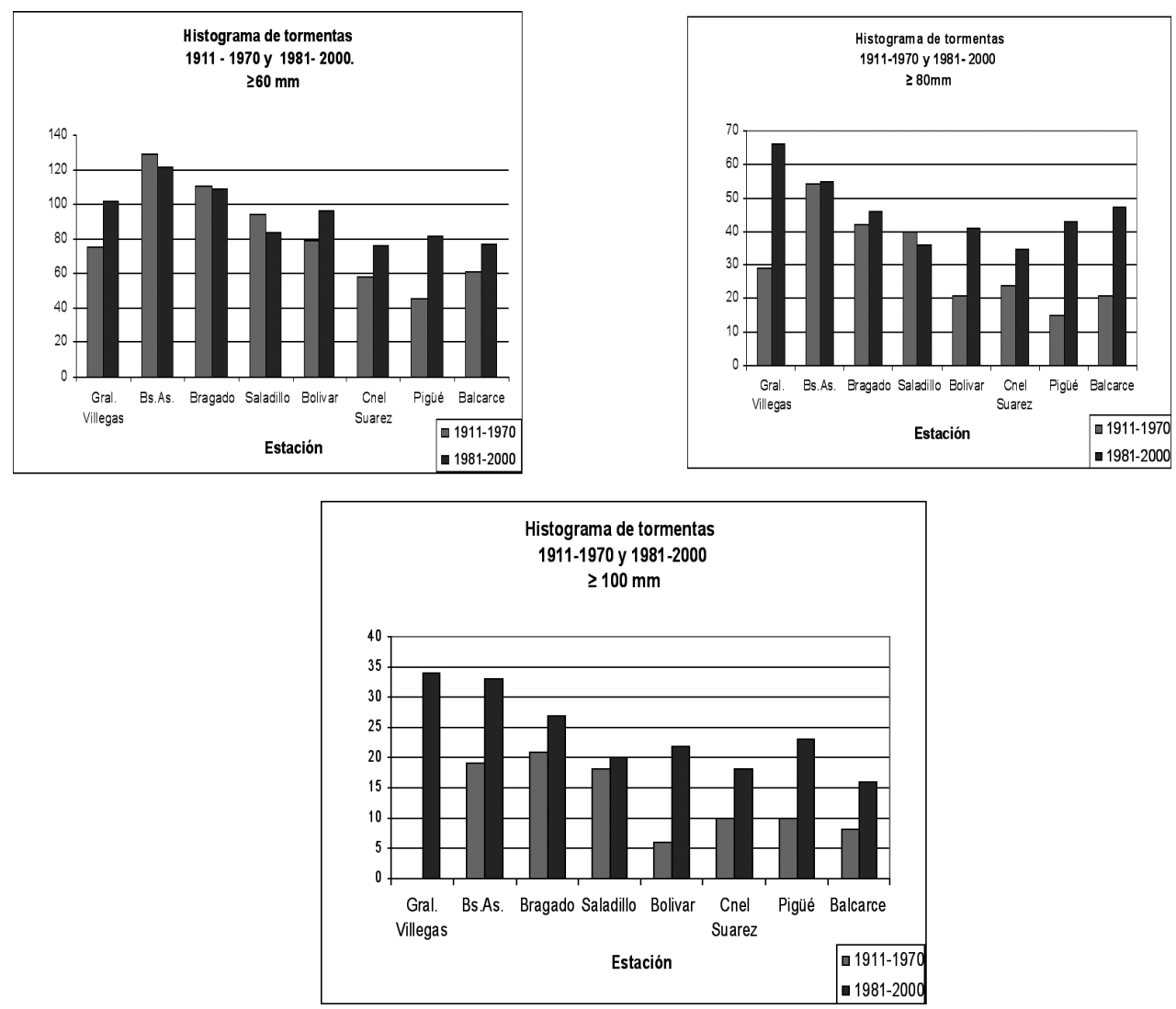

Figura 3. Histogramas de tormentas en la región de la Pampa bonaerense. Periodos 1911-1970 y 1981-2000 (Fuente: Canziani, 2003). 


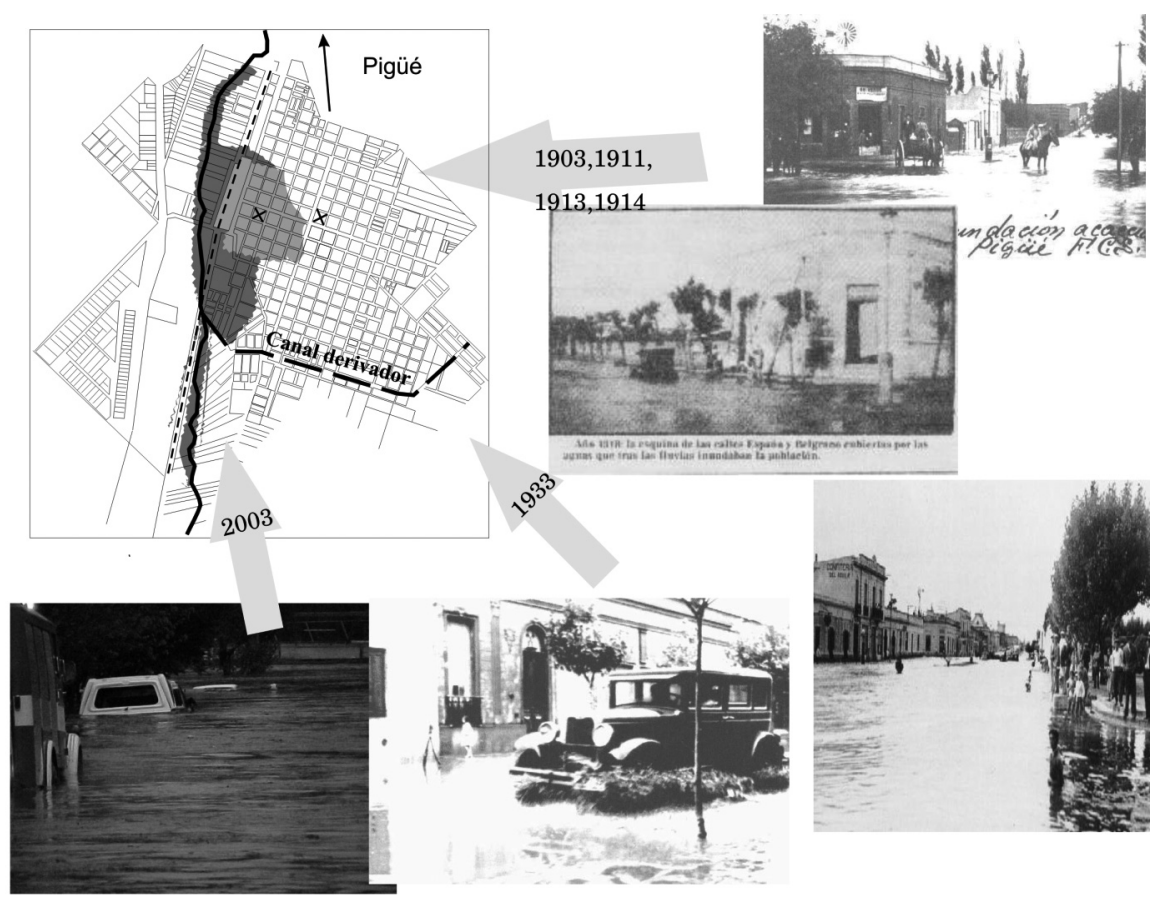

sector de riesgo de inundación desde 1914-2003

incorporación de nuevos sectores de riesgo de inundación 2003

Figura 4. Sectores del municipio con riesgo de inundación.

Sin embargo, su efectividad se vio comprometida el siete de octubre de 2003 cuando precipitaron, en dos horas y de forma simultánea, $152 \mathrm{~mm}$ en el casco urbano y 300mm en la cabecera; provocando así la mayor inundación de la historia de Pigüé (figura 4).

En este caso, la concentración y la presión de las aguas en el canal impidieron la libre evacuación del caudal del Pigüé, produciéndose importantes desbordamientos que anegaron el núcleo de población. Según crónicas locales, la lámina de agua alcanzó el metro y medio de altura en el centro de la ciudad, inundando 150 viviendas y la consiguiente evacuación de la población.

Surge pues, una vez mas, la alarma ante los eventos de avenidas, al comprobar la limitación del Canal Derivador para resolver los problemas de inundación del municipio. Por tanto, es de vital importancia resaltar la necesidad de abordar la prevención de los riesgos de inundación en la zona, no sólo desde una perspectiva estrictamente hidráulica, sino también desde un enfoque más de tipo ambiental (Zinger y Rosell 2004).

\section{Balance hídrico y comportamiento hidrológico de la cuenca}

Con el objetivo de aportar algunas claves al estudio hidrológico de la cuenca alta del Arroyo Pigüé, se procede a la elaboración del balance hídrico del suelo. Para ello, se ha 
contado con los datos aportados por las estaciones meteorológicas de Pigüé (1964-1980), Coronel Suárez (1981-1990) y Estancia La Celina (1991-2007). La primera y la tercera se encuentran localizadas en la cuenca alta del arroyo Pigüé; mientras que la segunda, se sitúa a pocos kilómetros del municipio, fuera ya del ámbito de la cuenca de drenaje. Con ello, se aborda una orquilla temporal de 43 años, que queda integrada, en su totalidad, en el ciclo definido como húmedo en la serie de precipitaciones.

Los tipos de suelos predominantes de la cuenca y sobre los que se realizan los balances hídricos son los Argiudoles, de textura franco-limosa. Estos suelos, localizados en el Piedemonte y las lomadas, son considerados los más aptos para el cultivo del cereal. Se caracterizan por presentar una buena estructura y una capacidad de campo estimada, según tablas de Thornthwaite-Matter (1957), en $200 \mathrm{~mm}$.

\subsection{Balance hídrico: estación Pigüé (1964-1980)}

Aún cuando se cuenta con una serie de 17 años para la estación de Pigüé, se ha estimado oportuno subdividir la serie en dos periodos (1964-1972 y 1973-1980) para así unificar, grosso modo, el rango temporal con el del resto de las series estudiadas (Coronel Suárez y La Celina), y estar en condiciones de contrastar el comportamiento del suelo en cada momento.

Los primeros ocho años registrados en la estación de Pigüé (1964-1971) corresponden al inicio del ciclo húmedo en la región pampeana y se define, principalmente, por la irregularidad en la precipitación.

En suelos argiudoles (figura 5), el balance queda definido por una recarga hídrica que se inicia en el mes de marzo y se extiende hasta el mes de septiembre, inclusive. En estos siete meses, el incremento de agua capilar contenida en el suelo experimenta una tendencia decreciente desde el mes de marzo hasta el mes de julio; a partir del cual, dicho valor se incrementará hasta alcanzar la capacidad del campo y en consecuencia la saturación del suelo, en el mes de octubre. A partir de este momento y hasta diciembre, en el que se inicia el periodo de déficit hídrico, el suelo mantiene las condiciones de saturación, generando un máximo de excedente hídrico en el mes de octubre, que alcanza el 23\% del agua que queda retenida en el suelo. En cuanto a las pérdidas que experimenta el suelo durante los tres meses en los que se detecta condiciones de déficit, éstas son máximas en los meses de enero y febrero, perdiendo el suelo algo más del $44 \%$ de su humedad al final de la etapa seca. Pese a ello, el suelo mantiene durante estos meses cierta humedad edáfica.

El segundo periodo (1972-1980), donde el comportamiento de la precipitación es mas regular, se detecta una ampliación en el periodo de recarga, que se extiende desde marzo hasta el mes de octubre, inclusive (figura 5). Al igual que observábamos en el balance anterior, en este periodo la tasa de infiltración refleja dos máximos, uno al comienzo y otro al final de la etapa de recarga edáfica. De esta manera, alcanzada la saturación del suelo en el mes de noviembre, el escaso excedente hídrico que se genera, tan sólo corresponde al 9\% del agua retenida en el suelo. Por otro lado, las pérdidas reales que experimenta el suelo se ajustan a los meses de verano, detectándose un escaso déficit hídrico tan sólo en los meses de enero y febrero. Ello determina que el suelo, al final del periodo estival mantenga más del $50 \%$ de la humedad que le permite su capacidad de campo.

En definitiva, los balances reflejan el protagonismo de la recarga hídrica en el suelo, estimada en siete u ocho meses; mientras que el periodo de escorrentía es a lo sumo, de dos meses. Por tanto, la disponibilidad de agua hacia los cauces de drenaje, por saturación de suelos, será máxima en los meses de octubre y/o noviembre, coincidiendo, grosso modo, con precipitaciones abundantes en dichos meses y estar saturado el suelo. 

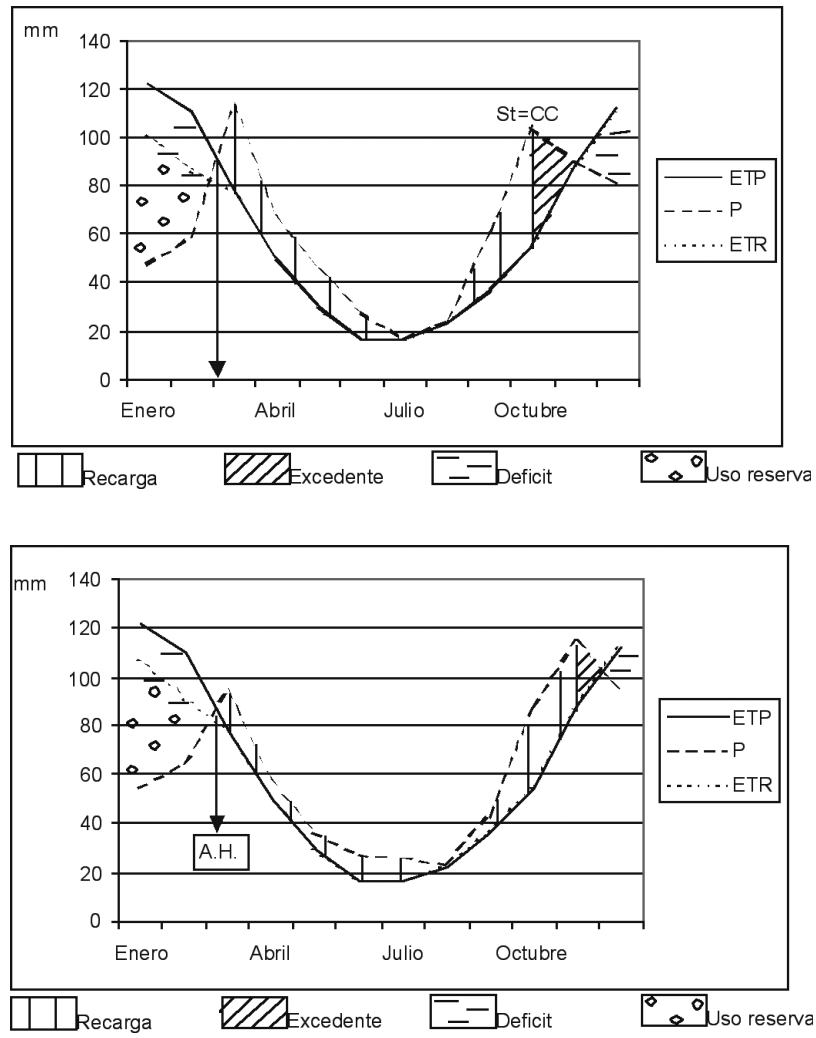

FiguRA 5. Balance hídrico para las series 1964-1971 y 1972-1980 (estación Pigüé)

\subsection{Balance hídrico: estación Coronel Suárez (1981-1990)}

Iniciado el año hidrológico en el mes de marzo, y con ello el proceso de recarga hídrica, el suelo alcanza su capacidad de campo en el mes de abril (figura 6). Dos meses son suficientes para que el suelo, en estas condiciones, alcance la saturación y dé comienzo el periodo de excedente hídrico, que se extenderá desde mayo a noviembre. Sin embargo, el comportamiento de las aguas de escorrentía no es homogéneo a lo largo de estos siete meses. Se detecta dos puntas, una en mayo y otra en octubre, coincidentes grosso modo con los valores máximos de precipitación. Igualmente se aprecia una etapa de déficit hídrico que se circunscribe a los meses estivales, aunque sin que se evidencie realmente problemas en la retención de humedad; puesto que el suelo, en este caso, tan sólo cede el 10\% del agua retenida en el periodo de adición de humedad.

A grandes rasgos, este comportamiento hídrico del suelo puede responder a dos situaciones distintas. Si comparamos los volúmenes de precipitación media mensual registrados en la estación de Coronel Suárez y en la de Pigüé, éstas últimas pese a ser mayores que las primeras, no generan importantes procesos de escorrentía superficial. Ello puede hacer pensar que las precipitaciones registradas en la estación Coronel Suárez, si bien son menores, pudieran 


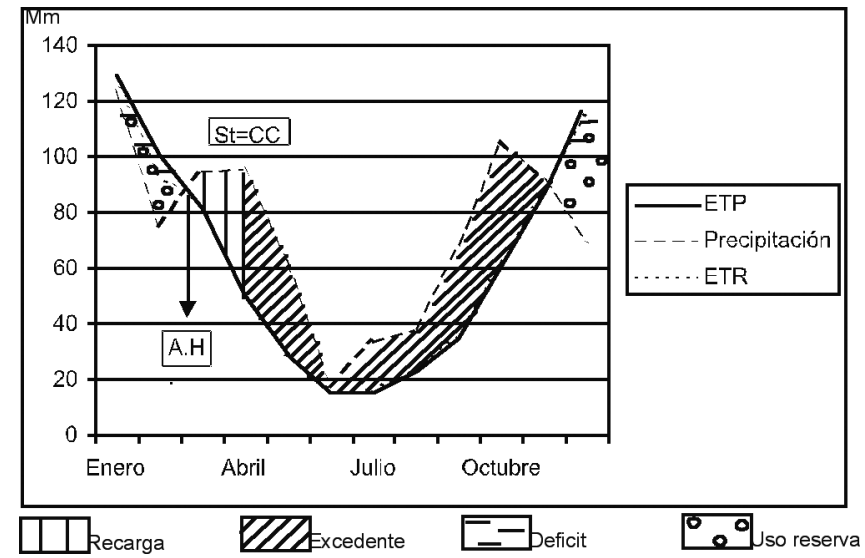

FIGURA 6. Balance hídrico para la serie 1981-1990 (estación Coronel Suárez)

haber sido de carácter tormentoso. En este caso, podría darse escorrentía superficial sin existir humedad edáfica. Sin embargo, no se tiene constancia escrita de que en este período haya existido inundaciones en el municipio de Pigüé. De no ser así, dicho comportamiento bien pudiera reflejar suelos con unas condiciones constantes de humedad, en los que los meses estivales no supusieran importantes pérdidas; por lo que la recarga se efectuaría muy rápidamente. Si observamos el periodo 1972-1980, según los datos de Pigüé, durante el verano el suelo mantiene más del $50 \%$ de la humedad que le permite su capacidad de campo.

\subsection{Balance hídrico: estación Estancia La Celina (Piedemonte Sierra cuenca del Pigüé)}

En el último decenio del siglo XX, cuando la tendencia de las precipitaciones marca su máximo en el ciclo húmedo de la serie estudiada, el balance hídrico del suelo muestra un comportamiento hídrico caracterizado por sus condiciones de saturación (figura 7). En este caso, ni siquiera el periodo estival supone un verdadero déficit, pues ni en estas circunstancias el suelo pierde más de una veintena de milímetros de humedad, lo que supone un volumen de retención de $183 \mathrm{~mm}$ al final del verano.

Esta situación de saturación del suelo, en el momento de máxima humedad, trae consigo un período de diez meses de excedente hídrico en el suelo, que se traduce en la generación de escorrentía en superficie y la transmisión de humedad por percolación hacia el acuífero. Su comportamiento experimenta una cierta variabilidad temporal, detectándose tres picos más o menos individualizados, sin que en ningún caso supere la centena de milímetros: el mayor al comienzo de otoño, concretamente en el mes de junio $(84.4 \mathrm{~mm})$, al que le sigue el mes de abril $(61.5 \mathrm{~mm})$ y el mes de noviembre $(32.4 \mathrm{~mm})$.

Por su parte, el comienzo del siglo XXI marca el nivel máximo de precipitación alcanzado en la serie estudiada de 120 años; concretamente en el año 2001 con un volumen de precipitación próximo a $1400 \mathrm{~mm}$. Sin embargo, le siguen otros que estarían por debajo de la precipitación media estimada para la zona $(750 \mathrm{~mm})$, concretamente el año 2003 con un valor anual de $600 \mathrm{~mm}$ y el 2006 por debajo de los $500 \mathrm{~mm}$.

En este contexto se detecta un balance hídrico caracterizado, igualmente por el nivel de saturación del suelo, aún cuando con ciertas diferencias respecto al anterior (figura 7). En 
este caso, el excedente hídrico que genera el suelo, como consecuencia de la saturación se concentra en el mes de octubre, con un total de $193 \mathrm{~mm}$. Esta concentración de agua en un solo mes, puede responder a precipitaciones de carácter torrencial, tan características en la zona, que unido a las condiciones de saturación descritas genera altos niveles de escorrentía. Junto a ello, se detecta un periodo de déficit hídrico muy débil en los meses de diciembre y enero, recuperando el suelo su capacidad de campo en el mes de marzo.

Es precisamente en este comienzo del siglo XXI cuando tiene lugar la mayor inundación registrada en Pigüé. Para contextualizar dicho evento se ha individualizado el balance hídrico de los años precedentes, esto es 2000-2003. De los tres balances analizados en la estación de La Celina, éste (figura 8) es el que podemos considerar más húmedo. Tal y como se aprecia en la gráfica, prácticamente no podemos hablar de periodo seco, puesto que tan sólo se detecta un escaso déficit en el mes de febrero, que tan sólo supone una pérdida en el suelo de $8 \mathrm{~mm}$, quedando el suelo con un volumen de agua capilar de 192 $\mathrm{mm}$, para el final del verano. En este sentido, en marzo se iniciaría la recuperación hídrica, consiguiendo rápidamente la saturación del suelo que se extenderá, incluso, hasta el mes
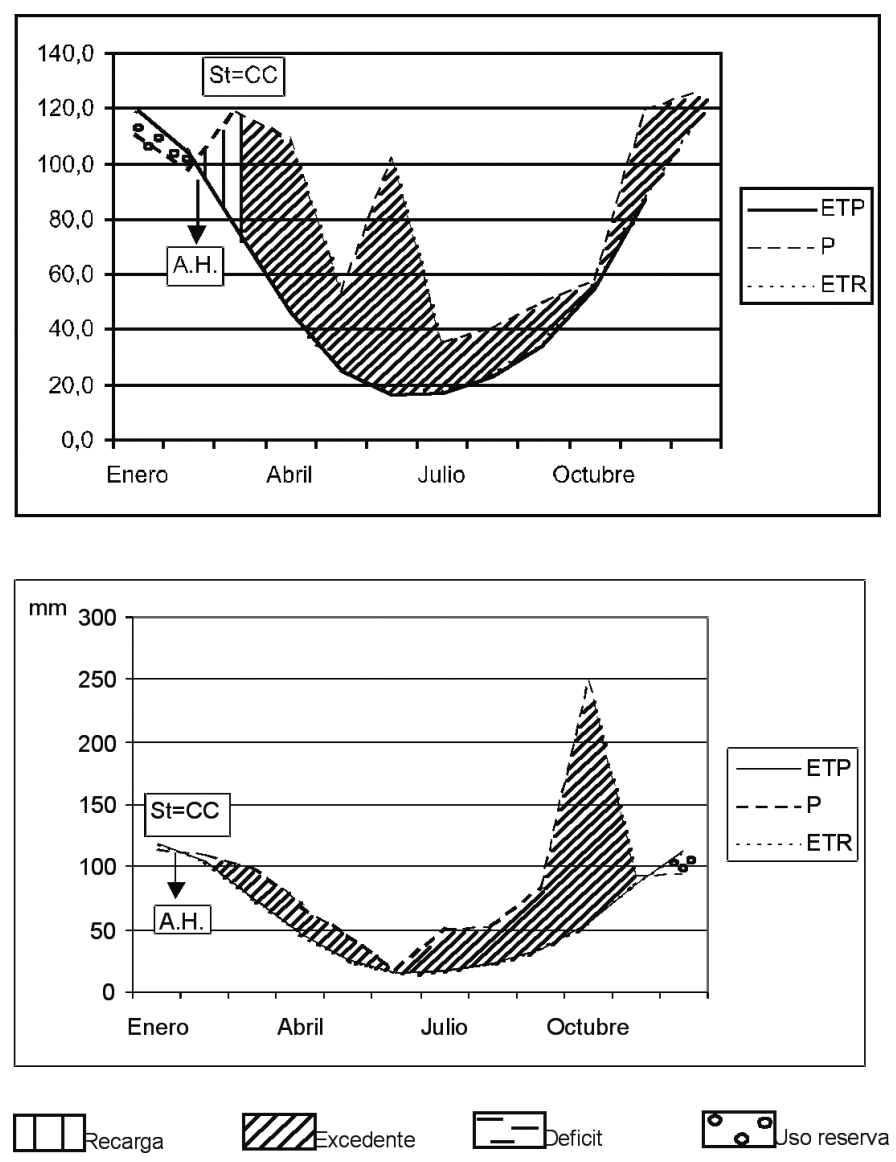

FiguRA 7. Balance hídrico para las series 1991-1999 y 2000-2007 (estación La Celina) 

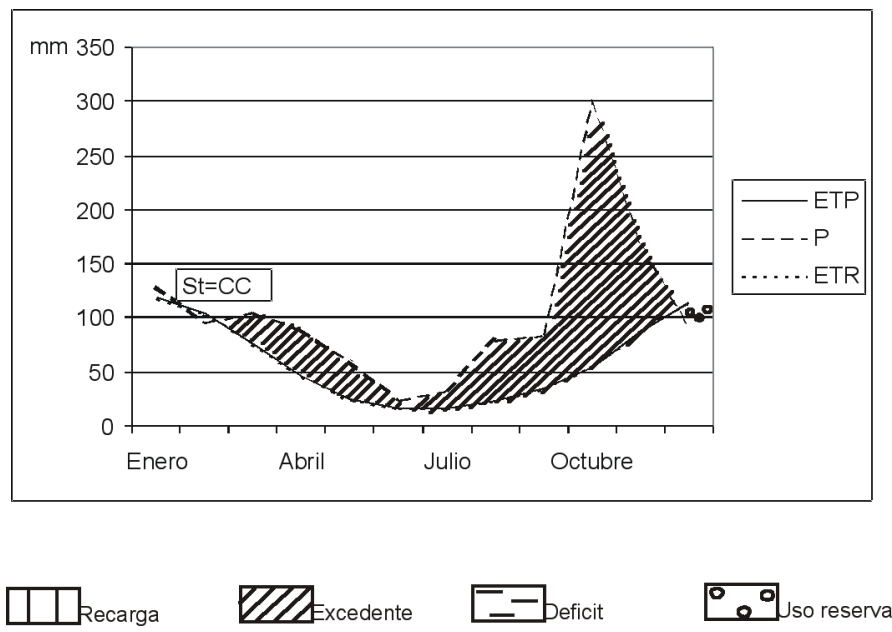

FiguRa 8. Balance hídrico para la serie 2000-2003 (estación La Celina)

de enero. Esta saturación genera un excedente hídrico sin precedente en los balances estudiados, alcanzando un máximo histórico en el mes de octubre, con un volumen de agua superior a los $240 \mathrm{~mm}$.

Según los datos aportados por la estación meteorológica, el catorce de octubre de 2003 tuvo lugar una tormenta en la que precipitó cerca de $300 \mathrm{~mm}$ en dos horas. El carácter tormentoso de esta precipitación, unido a la saturación del suelo descrita es lo que provoca la avalancha de caudal y aluviones hacia la localidad de Pigüé.

\section{Resultados y conclusiones}

El comportamiento hidrológico de la cuenca alta del Arroyo Pigüé, en la segunda mitad del siglo XX e inicios del XXI, es el resultado de la interrelación de una serie de factores que, de manera general, controlan los sistemas fluviales en cabecera; esto es, el clima, la vegetación natural y la antropización de los interfluvios.

Desde un punto de vista climático, en este período, se constata la existencia de un ciclo húmedo en la región, argumentado por un desplazamiento histórico de las isohietas hacia el Sur y el Oeste, a partir de 1970. Este hecho, que de manera general, trae consigo un aumento de los valores medios de precipitación anual en toda la provincia de Buenos Aires, se concreta en un aumento de $100 \mathrm{~mm} / \mathrm{año} \mathrm{y}$ en un incremento de las precipitaciones de carácter tormentoso en el Distrito de Saavedra, donde se localiza el municipio de Pigüé.

Junto a ello, en la cuenca alta del Pigüé son numerosos los signos que revelan una intensa antropización del sistema. El protagonismo del monocultivo del cereal frente a la vegetación natural, que queda restringida a partir de la cota de $400 \mathrm{~m}$, infiere una reducción en la capacidad de retención de los suelos, estimada para este caso en $200 \mathrm{~mm}$ en suelos Argiudoles. Este hecho, trae consigo la pronta saturación de los suelos en periodos húmedos que, unido a las pendientes propias de la zona, primarán la escorrentía superficial, con el consiguiente lavado de suelos y pérdidas de nutrientes. Así mismo, la rápida saturación de los suelos provocará una mayor disponibilidad de agua vertiente abajo; lo que podría agravar el riesgo de inundación en el municipio de Pigüé. 
Los balances hídricos elaborados en la cuenca a partir de las estaciones meteorológicas de Pigüé, Coronel Suárez y La Celina en la orquilla temporal 1964-2007, así lo demuestran. En condiciones normales de humedad, es decir, cuando el valor medio de precipitación ronda los 750mm, el balance hídrico muestra una recarga hídrica de siete u ocho meses a partir del mes de marzo, en el que se identifica el comienzo del año hidrológico. En estos casos, el periodo estival queda perfectamente definido a partir de la pérdida de agua del suelo, máxima en los meses de enero y febrero. Sin embargo, a medida que nos adentramos en el ciclo propiamente húmedo a partir de la década de los ochenta, el suelo va dando muestras de su incapacidad para retener agua, saturándose cada vez más rápidamente y primando la escorrentía superficial en dirección a Pigüé. Estos efectos son tanto más intensos cuanto mayor es el carácter tormentoso de la precipitación. El caso más extremo lo encontramos reflejado en el balance previo a la avenida registrada en octubre de 2003. Tras varios años húmedos en los que se mantiene el nivel de saturación del suelo, incluso en época estival, se le añade la descarga en cabecera de $300 \mathrm{~mm}$ en dos horas.

Por otro lado, es necesario destacar el desmantelamiento de gran parte de la red fluvial visiblemente desconectada en el territorio, como consecuencia de la intensificación de las prácticas agrícolas. En estas condiciones la escorrentía superficial generada se evacuará con mayor rapidez siguiendo las pendientes dominantes hacia el arroyo Pigüé, sin encauzamiento alguno y éste hacia el municipio; tal y como aconteció en la crecida del año 2003.

Hasta entonces, la construcción del Canal Derivador en el año 1952 había minimizado el riesgo de inundación en el municipio, produciéndose tan sólo inundaciones de menor envergadura y de carácter recurrente. Sin embargo, la avenida de 2003, en la que coincidió la saturación del suelo, en ciclo húmedo, con precipitaciones de carácter tormentoso, superó todas las previsiones. Queda así en entredicho la labor del Canal Derivador en condiciones extremas.

En este sentido, y teniendo en cuenta las condiciones climáticas actuales, sometidas a constantes cambios extremos, urge más si cabe, la necesidad de una ordenación integral a escala de cuencas vertientes. En la que no prime exclusivamente la perspectiva hidráulica, como la construcción del Canal Derivador para minimizar los riesgos de inundación en Pigüé; sino que se aborden así mismo otros aspectos más de tipo ambiental, en el que el uso del suelo y las practicas agrícolas sean aspectos claves en la ordenación. De esta manera se reduciría la rápida saturación de los suelos así como las tasas de erosión en periodos hidrológicos extremos.

\section{Referencias bibliográfícas}

BÁRDOSSY A., LEHMANN W. (1998): Spatial distribution of soil moisture in a small catchment. Part 1: geostatistical analysis. J. Hydrol., 206: 1-15.

CAMPO DE FERRERAS, A. y CAPELLI STEFFENS, A. (2004): El clima del suroeste bonaerense, Dpto. de Geografía y Turismo, Universidad Nacional del Sur, Bahía Blanca.

CANZIANI, P (2003): La tierra en peligro. Primer Seminario Internacional Cambio Climático y Sumideros de Carbono.

COSANDEY, C. et ROBINSON, M. (2007): Hydrologie continentale. Armand Colin, Paris, 360 p

FAMIGLIETTI J. S., RUDNICKI J. W., RODELL M. (1998): Variability in surface moisture content along a hillslope transect: Rattlesnake Hill, Texas. J. Hydrol., 210: 259-281.

GONZÁLEZ URIARTE, M. y ORIOLI, G. (1998): Carta geoambiental del Partido de Guaminí. Provincia de Buenos Aires. EDI UNS.

GRAYSON R. B., WESTERN A. W., CHIEW F. H. S., BLÖSCHL G. (1997): Preferred states in spatial soil moisture patterns: Local and non local controls. Water Resour. Res., 33 (12): 2897-2908. 
HOFFMANN, J., NUÑEZ, S. y GÓMEZ, A. (1987): Fluctuaciones de la precipitación en la Argentina, en lo que va del siglo. II Congreso Interamericano de Meteorología. V Congreso Argentino de Meteorología. Buenos Aires. Argentina.

INSTITUTO NACIONAL DE TECNOLOGÍA AGROPECUARIA (1971): Atlas de suelos de la República Argentina. Secretaría de Agricultura, Ganadería y Pesca de la Rca. Argentina e Instituto Nacional de Tecnología Agropecuaria. Buenos Aires.

KRUSE, E. y LAURENCENA, P. (2005): «Aguas superficiales. Relación con el régimen subterráneo y fenómenos de anegamiento.» Relatorio del XVI Congreso Geológico Argentino. La Plata Argentina.

ROSELL, P. y ZINGER, A. (2003): «Arroyo y ciudad de Pigüé: diagnóstico preliminar de una relación conflictiva» Revista Universitaria de Geografía, 12, n ${ }^{\circ}$ 1-2, Dpto. de Geografía.

SCIAN, B.; DONNARI, M. (1997): Retrospective analysis of the Palmer drought severity index in the semi-arid Pampas region, Argentina. Inter. J. Climatology, 17:313-322.

THORNTHWAITE, W. C. y MATTER, J.R. (1957): Instructions and tables for computing potential evapotranspiration and water balance. Publications in Climatology. Volume 3. New Jersey.

ZINGER, S. y ROSELL, P. (2004): «Inundaciones: importancia de un tratamiento integral. Caso: ciudad de Pigüé. Provincia de Buenos Aires». En Actas del $6^{\circ}$ Encuentro Internacional. Humboldt. Villa C. Paz. 